\title{
Design and implementation of a UWB six-port correlator for 6-9 GHz frequency band
}

Imran Mohsin, Magnus Karlsson, Owais Owais and Shaofang Gong

The self-archived postprint version of this journal article is available at Linköping University Institutional Repository (DiVA):

http:// urn.kb.se/ resolve?urn=urn:nbn:se:liu:diva-87198

N.B.: When citing this work, cite the original publication.

Mohsin, I., Karlsson, M., Owais, O., Gong, S., (2013), Design and implementation of a UWB six-port correlator for 6-9 GHz frequency band, Microwave and optical technology letters (Print), 55(1), 190193. https:// doi.org/ 10.1002/ mop.27235

Original publication available at:

https:// doi.org/ 10.1002/ mop.27235

Copyright: Wiley (12 months)

http:// eu.wiley.com/WileyCDA/ 


\title{
Design and Implementation of a UWB Six-Port Correlator for 6 to $9 \mathrm{GHz}$ Frequency Band
}

\author{
Imran Mohsin, Magnus Karlsson, Owais, and Shaofang Gong. \\ Linkoping University, Department of Science and Technology, SE-60174 Norrköping, Sweden \\ E-mail:imrmo116@student.liu.se
}

\begin{abstract}
A compact size six-port correlator utilizing bandwidth enhancing stubs for the 6 to $9 \mathrm{GHz}$ ultra wideband (UWB) radio is presented. The proposed six-port correlator is designed for a center frequency of $7.5 \mathrm{GHz}$ and has a relative bandwidth of $50 \%$. The correlator is based entirely on microstrip technology for low cost and simple circuit implementation.
\end{abstract}

Keywords - Branch line coupler, Six-port correlator, Ultra wideband, Microstrip technology, and Wireless networks.

\section{INTRODUCTION}

The six-port correlator has been used in different measurement applications since 1960s [1], [2]. Later in the early 1970s, this idea was further explored by Engen and Hoer [3]-[6], since then six-port correlators have been used in many different kinds of applications [7]. The six-port correlator technique has been receiving a lot of attention for many of its uses, especially because of its good performance in measuring the reflection coefficient, low cost, and low power consumption. Six-ports are widely use these days in microwave and wireless applications like radar, reflectometer, antenna measurement, receiver, transmitter, transceivers, ultra wideband system and many more [7].

In wireless communications, low power consumption is important, since the devices often are battery operated. As compared to active mixers commonly used in transmitters and receivers, the six-port correlator as a passive component is an interesting solution with less power consumption as well as with less complexity. This passive component can provide wideband and low cost transceivers by using power detector instead of active mixers [7]. The Federal Communication Commission (FCC) in February 2002 specified $7.5 \mathrm{GHz}$ of frequency spectrum for the ultra wideband (UWB) in the range of 3.1 to $10.6 \mathrm{GHz}$, first time for public use [8], [9]. After some restriction imposed by Europe, Japan and later by China on the 3.1 to $4.8 \mathrm{GHz}$ band, 6 to $8.5 \mathrm{GHz}$ band in Europe and 6 to $9 \mathrm{GHz}$ band in China become more interesting because of higher data rate [8]. Six-port radio is a good choice to use for 6 to $9 \mathrm{GHz}$ frequency band because of its efficient architecture that provides good performance in UWB transmitters and receivers [7].

In this paper, a UWB branch coupler and a six-port correlator are presented, which are designed at the center frequency of $7.5 \mathrm{GHz}$ having $50 \%$ relative bandwidth. These circuits are designed by using Agilent's Advanced Design System (ADS). The proposed UWB six-port correlator has reduced the total covered area as compared to those proposed in pervious papers [2], [10].
The conventional $90^{\circ}$ branch line coupler has a relative bandwidth of only up to $10 \%$ [11], [12]. The conventional branch line coupler with its simplest structure has a narrow bandwidth because of small flexibility available in its design parameters, so its bandwidth is limited [12]. Matching network (open end, single stub) between junctions and ports is used to broaden the impedance bandwidth. Coupling of a two branch coupler is independent of frequency, actually it depends only on the admittance of each port [13]. Then, it is clear that by using the suitable matching network, junction can be matched and required coupling (which need to stay same) can be achieved at any frequency [11].

The UWB six-port correlator is designed with three compact UWB hybrid couplers that have tuning stubs which are carefully bent towards each other to reduce the circuit area. The object of this paper is to design a six-port correlator for 6 to $9 \mathrm{GHz}$ (50\% relative bandwidth). Moreover a margin in frequency band is good against the uncertainties in the fabrication process. Especially against the error of band shifting that can come because of the difference in simulated and measured electrical lengths [14].

\section{SIX-PORT CORRELATOR}

Fig. 1 shows the block diagram of a six-port correlator with tuning stubs which consists of one power divider and three quadrature hybrid couplers. Here if we consider the ports P1 and $\mathrm{P} 2$ as input ports then the four other ports from port P3 to P6 are output ports and the last port P7 is terminated with 50 $\Omega$. If the correlator is used as a mixer, the local oscillator (LO) signal is applied at port P1 and the radio frequency (RF) signal is applied at port $\mathrm{P} 2$, then the output signals will be processed at ports (P3 to $\mathrm{P} 6)$.

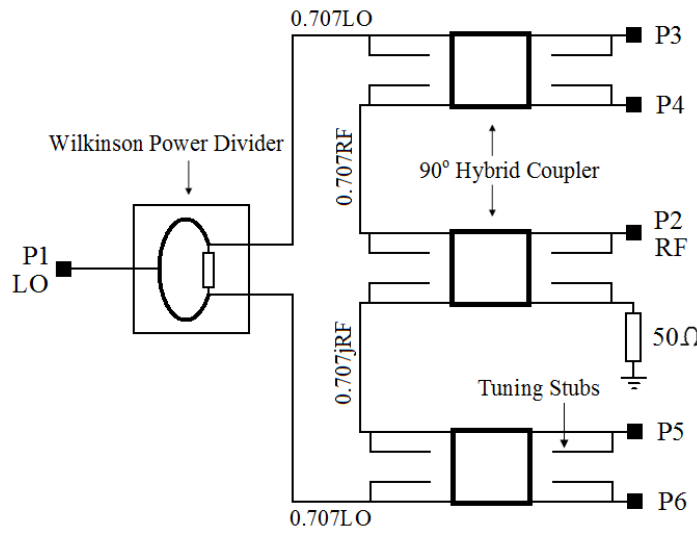

Fig. 1. Block diagram of six-port correlator. 
The Wilkinson power divider is a passive component that is used to divide incoming signal in to two equal signals. The phase difference between output ports of the power divider should be zero degree ideally. The Wilkinson power divider is a relatively broad band device that without any modification can operate in the whole desired bandwidth $(50 \%$ relative bandwidth) [10].

The quadrature $\left(90^{\circ}\right)$ hybrid coupler (also known as branch line coupler) consists of four ports. Unlike the power divider, the quadrature hybrid coupler is not a broad band device [10], [12]. For the frequency band of interest, return loss $\left(S_{11}\right)$, coupling $\left(\mathrm{S}_{21}, \mathrm{~S}_{31}\right)$ and isolation $\left(\mathrm{S}_{41}\right)$ should remain in certain limits but it is not necessary that circuit results follow perfect at all frequencies [15].

For the six-port correlator shown in Fig. 1, two important parameters to check the circuit performance are amplitude imbalance and phase difference between the output signals at port pairs P3-P4 and P5-P6. If ports P1 and P2 are considered as input alternatively, then the maximum amplitude imbalance between output ports should be less than $1 \mathrm{~dB}$ and the nominal phase difference should be $90^{\circ}$ [10].

\section{SIMULATED AND MEASURED RESULTS}

These circuits (UWB coupler and six-port correlator) are designed by using ADS simulator and manufactured on the double-sided Rogers 4350B printed circuit board (PCB) by using the microstrip technology. Measurements of the prototypes were done with the two ports Rohde \& Schwarz ZVM vector network analyzer. Table I summarizes the substrate parameters of the PCB.

TABLE I

SUBSTRATE PARAMETERS

\begin{tabular}{|c|c|}
\hline Dielectric thickness & $254 \mu \mathrm{m}$ \\
\hline Relative dielectric constant & 3.48 \\
\hline Dissipation factor & 0.004 \\
\hline Metal thickness & $25 \mu \mathrm{m}$ \\
\hline Metal conductivity & $58 \mathrm{MS} / \mathrm{m}$ \\
\hline Surface roughness & $1 \mu \mathrm{m}$ \\
\hline
\end{tabular}

To achieve the flat amplitude throughout the whole bandwidth, open circuit stubs were utilized. The six-port correlator contains three couplers, so by achieving one good compact and efficient coupler we can achieve a good compact six-port correlator.

Fig. 2 shows the UWB microstrip coupler prototype. The area of this UWB branch coupler is $31.0 \times 7.4 \mathrm{~mm}$. With this UWB branch coupler, $50 \%$ relative bandwidth is achieved which covers the frequency range from 5.6 to $9.4 \mathrm{GHz}$ with a center frequency of $7.5 \mathrm{GHz}$ that is enough to design a sixport correlator for 6 to $9 \mathrm{GHz}$.

It is found that the measured results have higher insertion loss than the simulated results and the frequency band is slightly shifted in the measured results. This higher insertion loss is likely a result of surface roughness [16], [17] since
ADS (2009) simulation does not take roughness into count [14]. The frequency band is little shifted toward lower frequency but still covering the whole target frequency band (6 to $9 \mathrm{GHz}$ ). The reason for this shift may come from phase velocity because the simulated phase velocity may come from higher as compared to the measured phase velocity [14].

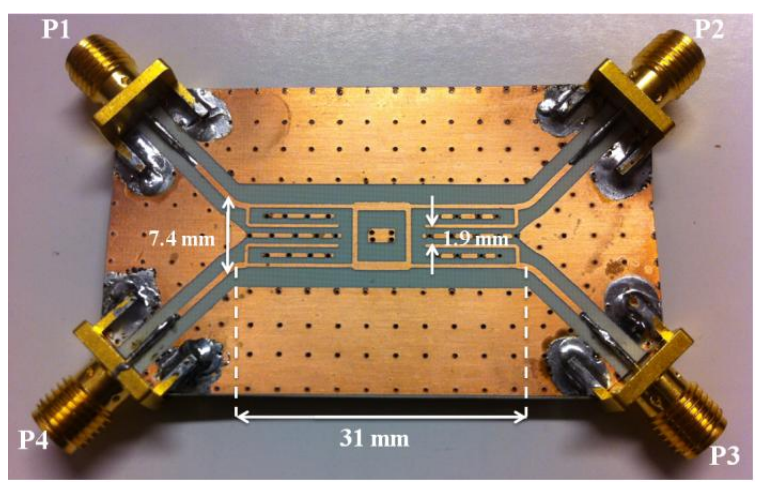

Fig. 2. Photo of UWB coupler prototype.

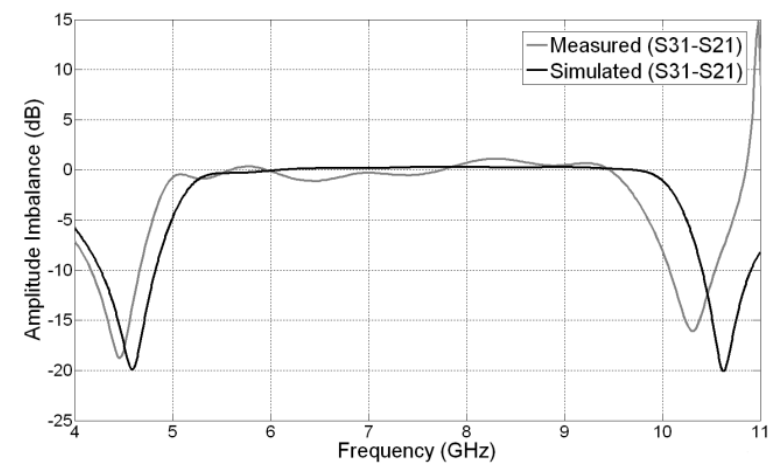

(a)

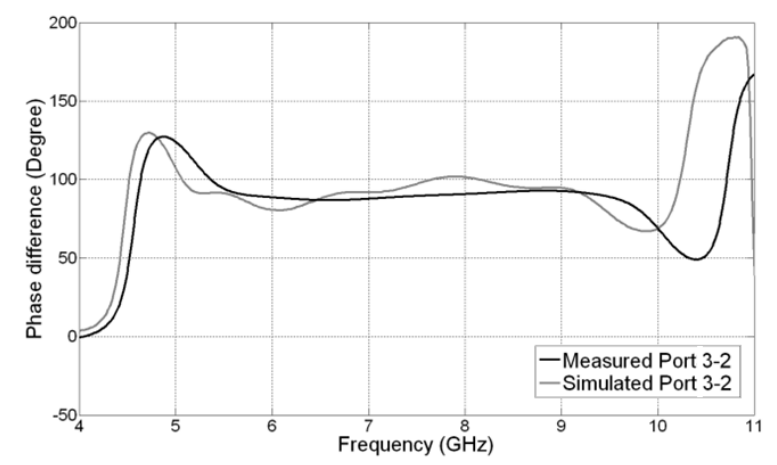

(b)

Fig. 3. Amplitude imbalance and phase difference of UWB coupler: (a) Amplitude imbalance, and (b) Phase difference.

As compared to the conventional branch line couplers, the UWB coupler (with the addition of the stubs) has a flat amplitude response and low coupling loss over a wide bandwidth. The UWB coupler has low insertion loss for the frequency range of 5.6 to $9.4 \mathrm{GHz}$. Simulated insertion loss varies from -3.3 to $-3.9 \mathrm{~dB}$, and measured one varies from 3.26 to $-4.80 \mathrm{~dB}$. From Fig. 3, it can be seen that amplitude 
imbalance of the coupler between output ports (P2 and P3) is $\pm 0.3 \mathrm{~dB}$ for its simulated and $\pm 1.1 \mathrm{~dB}$ for its measured results. As compared to the amplitude results of the coupler in paper [19] the coupler is slightly better. Supremely the phase differnce between the outputs of the coupler should be $90^{\circ}$. For UWB coupler, phase imbalance error for simulated results is varying from $-3.19^{\circ}$ to $2.81^{\circ}$ and for measured results from manufactured PCB is from $-9.61^{\circ}$ to $11.71^{\circ}$. For the measured results, amplitude imbalance are better than phase difference, in comparison from simulated results.

Fig. 4 shows the photo of the UWB six-port prototype. The actual circuit area of this six-port is $40.0 \times 28.8 \mathrm{~mm}$. Because of the size of SMA (SubMiniature version A) connectors, the line length of outputs and inputs are increased and bended.

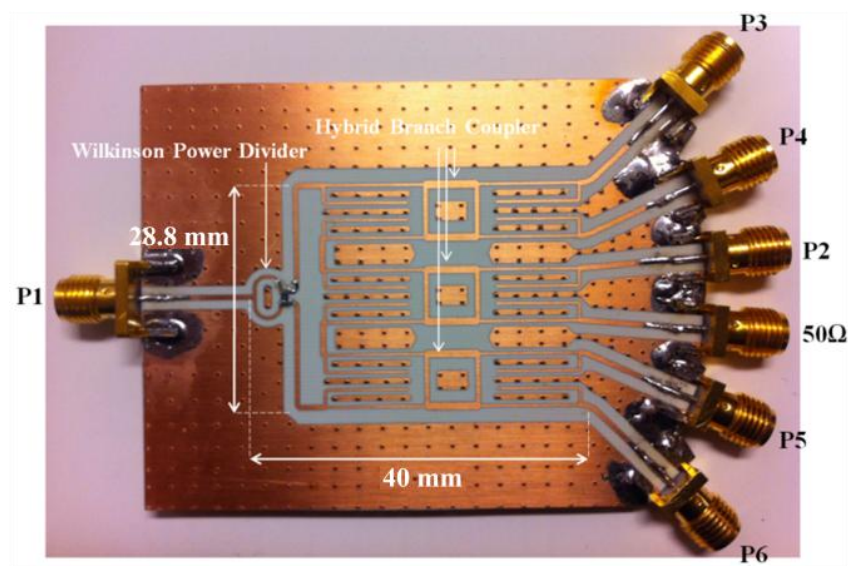

Fig.4. Photo of the UWB six-port prototype.

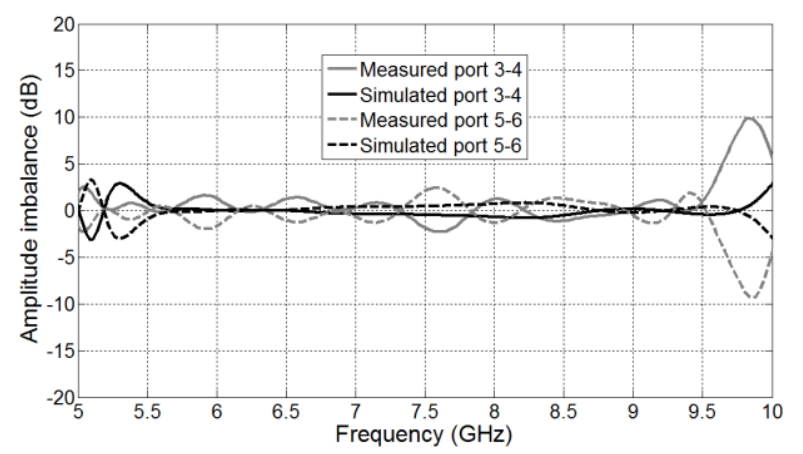

(a)

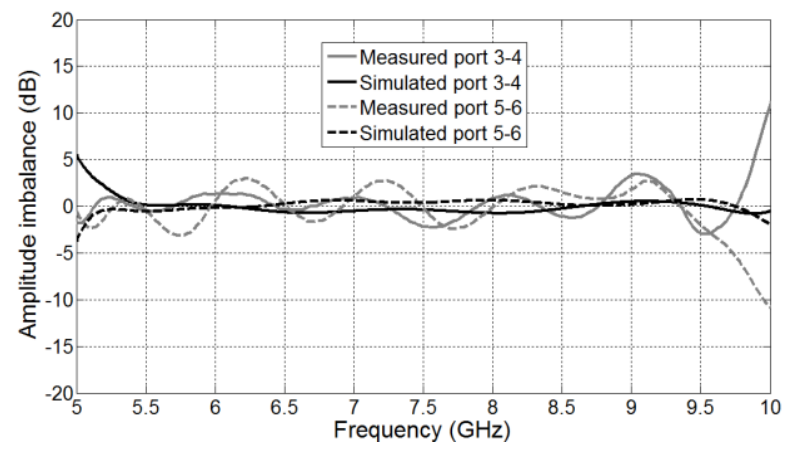

(b)

Fig. 5. Amplitude imbalance of UWB six-port: (a) Port 1 as input port, and (b) Port 2 as input port.

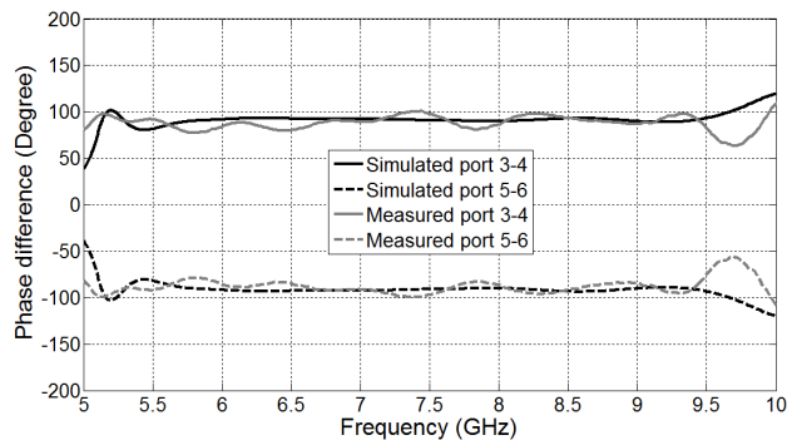

(a)

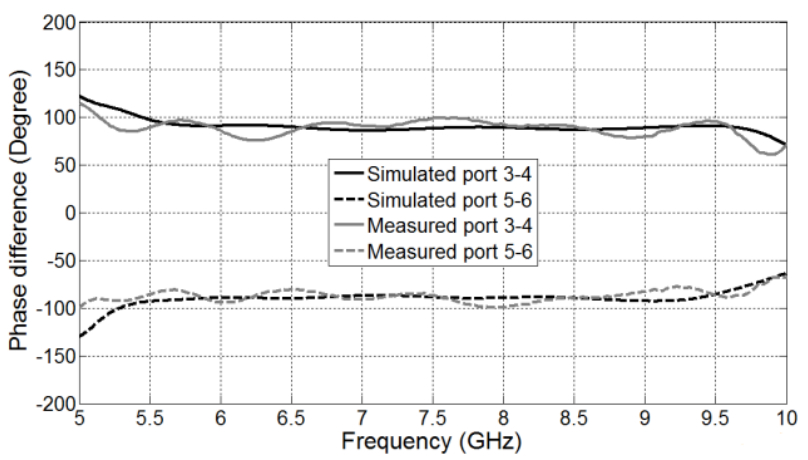

(b)

Fig. 6. Phase difference of UWB six-port:

(a) Port 1 as input port, and (b) Port 2 as input port.

Fig. 5 and Fig. 6 show simulated and measured results of the UWB six-port. In addition to a high relative bandwidth of this correlator, it has flat amplitude response for the frequency band from 5.4 to $9.4 \mathrm{GHz}$. This correlator has small insertion loss which varies from 6.4 to $10.1 \mathrm{~dB}$ for the frequency band of 5.6 to $9.0 \mathrm{GHz}$ and reaches to $13 \mathrm{~dB}$ at $9.4 \mathrm{GHz}$.

The maximum amplitude imbalance between output ports (P3-P4 and P5-P6) is limited to $0.82 \mathrm{~dB}$ and $2.90 \mathrm{~dB}$ for simulated and measured results, respectively. Input is applied alternatively to P1 and P2, see Fig. 6 (a) and (b) respectively. The maximum phase error (phase difference deviation) of this correlator from its nominal value of $90^{\circ}$ is $3.74^{\circ}$ and $11.20^{\circ}$ for simulated and measured results, respectively.

The difference between simulated and measured results is likely due to manufacturing, soldering, SMA connectors, and measurement accuracy errors [9]. During the design of the coupler and six-port a trade off is observed between the amplitude imbalance and phase difference error. In measured results, achieved amplitude imbalances are noticeably better than the maximum phase errors. References [10], [11], [13] show more concern about amplitude imbalances than phase error. However both the results should be considered together [19].

\section{DISCUSSION}

By bending the tuning stubs (open end, single stub) inside as in the UWB coupler, it takes $35 \%$ less area as compared to UWB coupler with tuning stubs bending outside. In the same manner a six-port correlator contains three couplers so it will 
affect more on the area. Bending of stubs inside towards each other need proper attention, because cross talk between stubs can occurs. The distance between stubs is $1.9 \mathrm{~mm}$ which is more than the twice of the width of the stubs and a ground plane is also present between stubs, these precautions are enough to secure signal quality and keep below cross talk to the acceptable level.

\section{CONCLUSION}

This paper presents a study on a UWB six-port correlator for the frequency band of 6 to $9 \mathrm{GHz}$. The branches of high impedance stubs in the UWB coupler enhanced its performance and the relative bandwidth is increased from $10 \%$ to $50 \%$. In order to deal with the design size issue, bending its stubs such that the size reduces to $31 \times 7.4 \mathrm{~mm}$. Another noticeable parameter is low insertion loss along with the flat amplitude imbalance for wide bandwidth. The maximum amplitude imbalances are \pm 0.30 and \pm 0.82 between 5.6 to $9.4 \mathrm{GHz}$ for the UWB coupler and six-port correlator respectively. Some occasional differences in the simulated and measured results of the UWB six-port correlator are observed due to substrate errors, soldering, SMA connectors and testing [10]. In general, the frequency band (6 to $9 \mathrm{GHz}$ ) is covered with a compact $(40.0 \times 28.8 \mathrm{~mm})$ low cost solution (based on microstrip technology).

\section{ACKNOWLEDGEMENT}

The authors would like to show deep respect and want to say special thanks to Mr. Gustav Knutsson at the Department of Science and Technology for his assistance in PCB manufacturing process.

\section{REFERENCES}

[1] S. B. Cohn and N. P. Weinhouse, "An automatic microwave phase measurement system," Microwave J., vol. 7, no. 2, pp. 49-56, Feb. 1964.

[2] A. Serban, J. Östh, Owais, M. Karlsson, S. Gong, J. Haartsen, and P. Karlsson, " Six-port Transceiver for 6-9 GHz Ultra wideband Systems," Microwave and optical technology letters, vol. 52 No. 3, March 2010.

[3] Hoer, C. A., "The Six-Port Coupler: A New Approach to Measuring Voltage, Current, Power, Impedance and Phase," IEEE Trans. IM, Vol. IM-21, 1972, pp. 466-470.

[4] Hoer, C. A., "Using Six-Port and Eight-Port Junctions to Measure Active and Passive Circuit Parameters,” NBS Tech. Note 673, 1975.

[5] Engen, G. F., "The Six-Port Reflectometer: An Alternative Network Analyzer," IEEE Trans. Microwaves Theory and Tech., Vol. MTT-25, pp. 1075-1079, 1977.

[6] Engen, G. F., "The Six-Port Measurement Technique-A Status Report," Microwave Journal, Vol. 21, pp. 18-89, 1978.

[7] F. M. Ghannouchi, and A. Mohammadi, "The Six-Port Technique with Microwave and Wireless Applications," Artech house, 685 Canton Street Norwood, 2009.

[8] A. Serban, "Ultra-Wideband Low-Noise Amplifier and Six-port Transceiver for High Speed Data Transmission," Thesis 1295, 2010.

[9] B. T. Ahmed and M. C. Ramon, "On the Impact of Ultra-Wideband (UWB) on Macrocell Downlink of UMTS and CDMA-450 Systems" IEEE Trans. Electromagnetic Compability, Vol. 50, no. 2, pp. 406-412, May 2008.

[10] D. Wang, A. Huynh, P. Häkansson, M. Li and S. Gong, "Study of Wideband Microstrip Correlator for Ultra-Wideband Communication System," Asia-Pacific Microwave Conference, 2007.
[11] R. Knochel, "Broadband flat coupling two-branch and multibranch directional couplers," IEEE MTT-s Int. Microw. Symp. Dig., 1990, pp.1327-1330.

[12] M. Muraguchi, T. Yukitake, Y. Naito, "Optimum design of $3 \mathrm{~dB}$ branch line coupler using microstrip lines," IEEE Trans. Microwaves Theory and Tech., vol. 31, pp. 674-678, 1983.

[13] G. P. Riblet, "A Directional Coupler with Very Flat Coupling," IEEE Trans. Microwaves Theory and Tech., vol. 26, pp. 70-74, Feb. 1978.

[14] M. Karlsson, P. Hakansson, S. Gong, "A Frequency Triplexer for Ultra-Wideband System Utilizing Combined Broadside- and EdgeCoupled Filters", IEEE Trans. On Advanced Packaging, vol.31, no.4, pp. 794-801, Nov. 2008.

[15] M. Muraguchi, T. Yukitake, Y. Naito, "Optimum design of $3 \mathrm{~dB}$ branch line coupler using microstrip lines," IEEE Trans. Microwaves Theory and Tech., vol. 31, pp. 674-678, 1983.

[16] X. Gu, L. Tsang, and H. Braunisch, "Estimation of roughness induced power absorption from measured surface profile data," IEEE Microwave Wireless Compon. Lett., vol. 17, no. 7, pp. 486-488, Jul. 2007.

[17] X. Chen, "EM modeling of microstip conductor losses including surface rougness effect," IEEE Microwave Wireless Compon. Lett., vol. 17 , no. 2, pp. 94-96, Feb. 2007.

[18] R. Knochel, "Broadband flat coupling two-branch and multibranch directional couplers", IEEE MTT-s Int. Microw. Symp. Dig., 1990, pp.1327-1330.

[19] D. Wang, A. Huynh, P. Häkansson, M. Li and S. Gong, "Study of Wideband Microstrip $90^{\circ} 3 \mathrm{~dB}$ Two-Branch Coupler with Minimum Amplitude and Phase Imbalance," IEEE ICMMT. Microwaves and Millimeter Waves Technology, vol. 1, pp 116-119, Apr. 2008. 